\title{
Experimental investigation of radiation transmission through a
}

\section{water spray}

\author{
G. Parent, P. Boulet*, S. Gauthier, J. Blaise and A. Collin \\ (* Corresponding author) \\ Laboratoire d'Energétique et de Mécanique Théorique \& Appliquée (LEMTA), CNRS UMR 7563 \\ Faculté des Sciences et Techniques BP 239 - 54506 VANDOEUVRE Cedex \\ Tel \& Fax (33) 383684686 - mail Pascal.Boulet@lemta.uhp-nancy.fr
}

Keywords : Transmissivity, radiative transfer, absorption, scattering, water spray

\begin{abstract}
Radiation attenuation by a water spray is experimentally investigated. Spectral transmissivity measurements are performed between 1000 and $7000 \mathrm{~cm}^{-1}$ with an experimental device involving a Fourier transform spectrometer. The spray is produced by a so-called Tee-Jet 400067 nozzle for water pressure between 1.5 and 6 bars. Key features like mean attenuation levels due to absorption and scattering by droplets and complex absorption pattern by water vapor are identified. Known effect of attenuation modification when increasing the water pressure is observed. A simulation is also performed to evaluate a numerical code developed in a companion study. The achieved agreement demonstrates the ability of the simulation to describe the radiation attenuation by the spray.
\end{abstract}

\section{Introduction}

Water sprays are well known as potential tools to attenuate radiation, for example radiation emitted by flames. Some protection devices are consequently developed using this ability. Experimental investigations aimed at the characterization and the optimization of such safety systems are reported in the literature (see for example in the last decade Prétrel [1], Dembélé et al. [2], Murrell et al.[3], Hald and Buchlin [4] or Widmann and Duchez [5]). Typical setups involve fluxmeters and comparisons of wall fluxes measured in the presence of the spray or not. However, few studies are reported on spectral 
measurement, although the attenuation due to the droplet pulverisation strongly varies with the incident wavelength of the radiation. Our goal is to restrict our analysis to fire protection using water curtains, radiation shielding being separated from the fire extinction problem. In this field, the reference study has been reported by Dembélé et al. ([2]), as a part of a complete work dedicated to radiation attenuation thanks to water curtains, a project named ASTRRE (in the frame of a European collaboration between research groups involving the CETHIL (Centre de Thermique de Lyon) and the VKI (Von Karman Institute - Brussels) among others).

Beside this work, recent reviews by Grant et al. [6] and Sacadura [7] essentially mention the above-cited test facilities based on flux measurements. The reported experiments contribute to characterize potential devices and nozzle abilities to yield sufficient radiation attenuation. Capability of the nozzles are also generally estimated in terms of water consumption. The contribution by Grant et al. [6] brings an extensive review of definitions and data related to sprays in the frame of fire suppression. We are rather concerned with the characterization of a device devoted to provide a shield between flame and a given target, but located sufficiently far from the fire area to be considered as a spray of droplets in wet air. No coupling with combustion or extinction process is to be addressed in the present study. Nevertheless, useful information may be also found in the literature dedicated to combined fire protection and extinction. As an example, the study by Grosshandler et al. [8], although concerned with extinguishing efficiency of room fire, provides and discusses velocity and size measurements obtained thanks to Phase Doppler Particle Analyser. In the frame of studies restricted to fire shielding, other characterizations of the hydrodynamics of sprays (droplet size distributions and velocities) have been reported. Through a series of studies carried out at the Von Karman Institute, Prétrel [1] or Zimmer [9] are for example recent contributions to a complete review of the available experimental data on the spray dynamics. Corresponding data may be consequently useful to understand the typical behavior of a given spray, even if they do not indicate the radiation attenuation ability in a direct manner in conditions close to our case study.

Our group at the LEMTA is involved in the simulation of radiation attenuation by water curtains. A numerical contribution has been first engaged, solely dedicated to the hydrodynamics of the spray and the coupling between turbulence and the droplet flow. This has been achieved in the frame of a collaboration between the LEMTA and the VKI (Zimmer et al. [9]). A complete coupling with radiation and energy balance is now in course. What is guiding us in the present study is the need for information on the spectral behavior of available nozzles and for data aimed at the validation of a numerical code devoted to the prediction of water curtain attenuation ability. Therefore, a device based on the experiment reported in [10] has been developed. The basics of the measurement process are similar, but the water feed involves an open loop (allowing the use of additives in water in the future, with easy cleaning of the device) and the Fourier transform spectrometer and 
the detector are up-to-date devices.

In the following, the experimental setup will be first detailed, then the measurement process will be presented. Results will be given on the characteristics of the tested nozzle, then a comparison with a numerical prediction of the radiation attenuation will be discussed.

\section{Experimental setup}

A complete view of the experimental device is presented on Figure 1. As it can be seen, the incident radiation leaves the spectrometer, crosses the spray and the transmitted signal is finally directed toward the detector and post-processed. The different main parts of the setup will be detailed hereafter.

[Figure 1 about here.]

\subsection{Water feed}

Figure 2 is a sketch of the part of the device devoted to the water feed of the nozzle. A technical solution warrantying a rigorous stability of the pressure in front of the nozzle has been chosen, involving pressurized air to compress water. A tank of air at a pressure around 8 bars is pressurizing water in the water feeder which has a maximum capacity of 10 liters. This is sufficient to allow the feed of the nozzle during 20 to 40 minutes depending on the liquid rate, which is low with the kind of laboratory nozzle used in the present study (namely from 0.26 to $0.50 \mathrm{~L} \cdot \mathrm{min}^{-1}$ in the present pressure range). Water is released thanks to a gate at a pressure measured by a gauge giving the water pressure just before the nozzle with a reading accuracy of 10 mbars.

[Figure 2 about here.]

\subsection{Spectrometer and detector}

A Fourier Transform InfraRed spectrometer (FTIR) (IFS66v/s type by Bruker) is used. This spectrometer is built around a Michelson interferometer and can be used under vacuum conditions, although this ability is not necessary here, the spray being in free space. The beamsplitter is a germanium on $\mathrm{KBr}$ one and the source is a globar. In this configuration its spectral range is $7500-500 \mathrm{~cm}^{-1}$. The movable mirror can be translated up to a speed that leads to an interferometric signal at a frequency equal to $100 \mathrm{kHz}$ with the radiation of He-Ne laser that controls the movement of the mirror. This maximum moving speed is used here as the detector has a sufficient bandwith $(16 \mathrm{MHz})$ to follow the obtained signal. That 
leads to a minimal acquisition time for an interferogram, so that a large number of acquisitions (scans) can be performed in order to decrease noise by averaging scans. The collimated beam exit of the spectrometer is used for the experiment. A MCT detector, liquid nitrogen cooled, by Kolmar technologies, working in photovoltaic mode insuring a good linearity is used. It's maximal spectral bandwidth is $12000 \mathrm{~cm}^{-1}-850 \mathrm{~cm}^{-1}$. Given the spectral bandwidth of the beamsplitter and of the detector, results presented here will be in the $1000-7000 \mathrm{~cm}^{-1}$ domain, as signal outside this range is quite small.

\subsection{Measurement process}

Our device is designed to provide direct spectral transmissivity data, measured through a spray irradiated by a collimated beam. Actually, our analysis of the optical path along which the beam is traveling yields the following angular characteristics:

- the spray is irradiated by a beam according to a solid angle around $3 \times 10^{-5} \mathrm{sr}$ and angular divergence around $0.35^{\circ}$ (as will be confirmed hereafter by the analysis of figure 7)

- the solid angle of detection is equal to $8.7 \times 10^{-6} \mathrm{sr}$ and angular acceptance is equal to $0.17^{\circ}$

Measurements are done with a $2 \mathrm{~cm}^{-1}$ spectral resolution. Each spectrum is the result of the Fourier transform of an average interferogram obtained with 500 scans. Moreover to avoid hazardous errors, typical measurements are repeated around 10 to 15 times in successive measurement sessions and averaging is performed on the obtained spectra. Despite the care brought to the design of the pressurized feed system, this method is applied to limit the uncertainty due to possible fluctuations in the spray production. Therefore the goal is to characterize the mean behavior of the nozzle regarding its attenuation efficiency whatever possible various surrounding conditions (temperature and moisture potentially affecting the transmissivity pattern).

Measurements can be carried out at different vertical and lateral positions from the nozzle and for several water feed pressure values. A series of acquisition processes consists in fixing the vertical and the lateral positions of the nozzle and then varying the feed pressure from 1.5 bars to 6 bars without modifying the nozzle position to avoid spray alignment alteration. Water tank is fed just before measurement, at surrounding conditions. The nozzle is first opened during a few minutes to stabilize the surrounding moisture and avoid an increase in the air moisture during the first stage of the measurement. 
Reference measurements without spray are performed immediately before and after each measurement through the spray and the reference spectrum used is the average of these two spectra in order to compensate a potential drift of surrounding conditions during the measurement with the spray on. Measurements of the transmissivity on the same optical path but with spray on are then carried out and ratio of transmission with the spray on over the case spray off is computed, as in relation (1). Such results will be presented in the next paragraph.

$$
T r=\frac{\text { Transmission acquisition with the spray on }}{\text { Transmission acquisition with the spray off }}
$$

\section{Results}

\subsection{Nozzle characterization}

The nozzle used for this study is referred as Tee-Jet 400067 (from Spraying Systems Co). The spray produced is a flat one, with a conic shape and an elliptic transversal section, the transmissivity being studied along the small axis. The cone angle according to the large axis is in the range $24^{\circ}$ at 1.5 bars to $53^{\circ}$ at 6 bars. The variations of nozzle water flow rate with the pressure of the water supply are shown in figure 3. Actually, the flow is known to increase with the square root of the pressure (see [8] or [9] for example) and one defines a so-called flow number that is equal here to $10.8 \mathrm{~L} \cdot \mathrm{min}^{-1} \cdot \mathrm{Pa}^{-1 / 2}$.

[Figure 3 about here.]

As it was said in the measurement process section, data recordings are repeated in successive sessions. They are done several days and several times a day for each combination of parameters that are adjustable in the experiment: water pressure $P$, vertical position $h$ of the measurement area down to the nozzle and lateral position from the axis of the spray. As a result, for a given set of parameters in the hereafter presented data, standard deviation is less than $0.5 \%$ outside the water vapor and carbon dioxyde absorption bands and around $1 \%$ inside them, leading so to an error less than $1 \%$ (in the $98 \%$ confidence interval) outside the absorption bands.

Spectral variations of the transmissivity are shown in figure 4 for five different water pressures, at $20 \mathrm{~cm}$ under the nozzle exit.

[Figure 4 about here.]

For a given water pressure, a rather flat transmissivity is observed with absorption bands due to a larger moisture in the spray than in the free air and a small slope of the transmissivity which increases with the wavenumber. A transmissivity 
decrease is also seen as water pressure increases, as we can expect it because the attenuation of the spray should be as a first approximation proportional to the volume fraction of water. More accurately, for a given size distribution of the droplet in the spray, absorption and scattering coefficients are proportional to the droplet number density. In conditions where transmission level is very high, outside water vapor absorption bands, attenuation is then proportional to the extinction coefficient $\sigma_{\mathrm{e}}=\kappa_{\mathrm{a}}+\sigma_{\mathrm{d}}$. As it was seen above, the water flow is proportional to the square root of the water pressure and then the attenuation would be proportional to the square root of the water pressure also. We will see in figure 5 , which presents the average transmissivity integrated over the whole wavelength spectrum as a function of pressure, that it is not exact. Actually, the transmissivity does not decrease below a minimum level as some other parameters, cited in the following items, play a role in the measured attenuation.

- The size distribution of droplets is varying when the pressure increases : Sauter mean diameter of droplets $d_{32}$ decreases when the pressure $P$ increases $\left(d_{32} \propto P^{-1 / 3}\right.$ from Prétrel [1]) and smaller droplets are more efficient regarding radiation absorption or scattering.

- On the contrary, the angle of the spray increases with pressure and leads to a lower quantity of droplets in the control volume than if this angle would be constant and then one have a smaller attenuation than expected.

- Moreover, the injection speed $v$ of the droplets increases with the pressure $(v \propto \sqrt{P}$ from Prétrel [1]) so that for a given flow rate the volume fraction of water and consequently the droplet number decreases.

- Finally, in single scattering mode, attenuation involves the extinction coefficient in an exponential term (BeerLambert's law) and is not linear with $\sigma_{\mathrm{e}}$.

In figure 5, the variations of the spectral averaged transmissivity with the water pressure are also presented for two other vertical positions in the spray. The attenuation is seen to be roughly the same whatever the vertical position as all curves are in the range of uncertainty, the singular behavior of the $40 \mathrm{~cm}$ curve being probably unrepresentative of an actual influence of the distance apart from the nozzle. As discussed above, the first stage of attenuation increase (transmission decrease) with pressure is obvious, whereas this trend is seen to stop as pressure reaches 4 bars. Note that this observation would probably be no more valid in a multiple nozzle device, spray broadening being potentially balanced by spray overlapping.

[Figure 5 about here.]

In order to study the variation of the transmissivity with the lateral position in the spray, a 18 mm diaphragmed beam is used rather than the free beam (around $40 \mathrm{~mm}$ diameter) that was used before. Results are shown in figure 6 . We see in 
this figure that for a zero lateral position $(x=0 \mathrm{~cm})$ the transmissivity obtained with the reduced beam is smaller than with the free beam whereas it is larger with a $2 \mathrm{~cm}$ shifted position. This clearly shows that optical and then other physical characteristics of the spray are not uniform on a $4 \mathrm{~cm}$ diameter area. Probably, a $18 \mathrm{~mm}$ diameter beam is still too large to obtain an actual local measurement but it is difficult to work with a smaller beam keeping enough signal and without having significant "free" scattering (scattering by an aperture) of the beam especially in I.R. for quite small wavenumbers. In fact, the measurement with the free beam is a kind of average on the measurement area. When shifting a bit over from the spray centre, at the $4 \mathrm{~cm}$ lateral position, transmissivity is still larger and then attenuation is smaller. This is due to a weaker droplet concentration and/or larger size droplets, which are known to be less effective regarding radiation attenuation when keeping the same water volume [11].

[Figure 6 about here.]

Let us now discuss some results regarding scattering effects. In figure 7 the normalized angular variations of the intensity detected with the spray on have been plotted versus the wavenumber and a detection angle. The reference measurement used for the normalization has been carried out with the detector centered according to the main propagation direction, where the intensity is the highest (reference angle value of $0^{\circ}$ ). Then the detector has been moved laterally and the detection angle $\varphi$ has been evaluated as $\varphi=\delta / f^{\prime}$ (where $\delta$ is the lateral displacement of the detector and $f^{\prime}$ the focal length of the spherical mirror used in order to focus light on the detector). The intensity is seen to decrease very quickly when shifting from the reference angular position, whereas a strong scattering influence would result in a less sharp pattern. Moreover, the angular width of the beam is seen to be rather constant when the wavenumber is varying and is around $0.18^{\circ}$ (half width). The same measurements with the spray off have given us very close results and therefore are not shown here in the same form. Some differences due to scattering are obtained only in the area of very low intensity. Scattering thus seems to have very little visible effect on the angular width of the beam. The explanation lies in the phase function, which exhibits an acute forward scattering as reported in Berour et al. ([12]).

\section{[Figure 7 about here.]}

In order to highlight the very thin differences between the beam with and without the spray the corresponding intensities in these two cases are shown in figure 8 for an incident wavenumber of $3000 \mathrm{~cm}^{-1}$ (the same comments would hold whatever the wavenumber of interest in the range studied here). Each measured intensity is normalized by its value obtained in the direct transmission direction $\left(0^{\circ}\right)$ and is plotted in a logarithmic manner as a function of the detection angle. Obviously, a small deviation exists between the two curves demonstrating that scattering occurs. However, it has to be emphasized that 
the beam measured with the spray on is really weakly larger and that scattering does not induce an important deviation from the propagation direction. Scattering influence will be discussed again in the numerical result discussion.

[Figure 8 about here.]

\subsection{Simulation and comparison with a numerical prediction}

The case of a vertical position $20 \mathrm{~cm}$ under the nozzle, at a pressure of $1.5 \mathrm{bars}$ has been chosen, since data for the size distribution have been given by the VKI in this particular case. The model used has been widely described in a recent paper and is not recalled here (see Collin et al. [11] for the full details of the following items). Main features are only given hereafter.

- Radiative transfer is addressed uncoupled from the rest of the spray modeling in a 1D stationary simulation, assuming azimuthal symmetry.

- The radiation source is approximated as a blackbody at temperature $1573 \mathrm{~K}$.

- A priori fixed data are introduced for the temperature: surrounding conditions evaluated at $300 K$, whereas an assumption of a cold medium is considered inside the spray, since the experimental device based on FTIR technology avoids any reinforcement contribution due to radiation emission (which is not coherent with the incoming radiation and does not contribute to the interference pattern). The water vapor volume fraction is evaluated assuming $60 \%$ of relative moisture when the spray is off and $80 \%$ when the spray is on. The curtain width is assumed to be $8 \mathrm{~cm}$, in accordance with the data introduced below for the size distribution, the whole set of conditions warrantying that the correct water quantity is taken into account. Moreover, this width value has been confirmed by direct observations of the spray at this vertical position, visualising a He-Ne laser beam usually of use to ensure the beam alignment through the spray.

- Size distribution is definitively fixed with assumed negligible size variations. The number density has been introduced as a function of droplet size for the different considered classes. Actually a Rosin-Rammler distribution has been introduced that best corresponds to the measured data. The mean Rosin-Rammler diameter has been found to be $245 \mu \mathrm{m}$, whereas the dispersion parameter is 2.64. Supplementary information for the spray is an averaged volumetric fraction of $5.85 \times 10^{-5} \mathrm{~m}^{3}$ of water $/ \mathrm{m}^{3}$ of air and a Sauter diameter of $182 \mu \mathrm{m}$. These data come from a specific study by L. Zimmer [13], dedicated to the present nozzle. Measurements were carried out using a PTVS technic (Particle Tracking Velocimetry Sizing). Data have been only corrected to correspond to the present 
exact flow rate, which has been measured to $0.2575 \mathrm{~L} \cdot \mathrm{min}^{-1}$ before transmission measurement. Finally the size distribution involved in the computation fulfills the actual flow rate and fits the experimentally observed distribution through a split in 49 diameter classes from 60 to $400 \mu \mathrm{m}$.

- The numerical solution is based on the Radiative Transfer Equation (RTE) written in a differential form as follows:

$$
\begin{array}{r}
\frac{\partial I_{\lambda}(s, \vec{\Omega})}{\partial s}=-\left(\kappa_{d \lambda}+\kappa_{c \lambda}+\sigma_{d \lambda}\right) \cdot I_{\lambda}(s, \vec{\Omega})+\left(\kappa_{d \lambda}+\kappa_{c \lambda}\right) \cdot I_{b \lambda}(T(s))+ \\
\frac{\sigma_{d \lambda}}{4 \pi} \int_{\Omega^{\prime}=4 \pi} P_{\lambda}\left(\overrightarrow{\Omega^{\prime}} \rightarrow \vec{\Omega}\right) \cdot I_{\lambda}\left(s, \overrightarrow{\Omega^{\prime}}\right) \cdot d \Omega^{\prime}
\end{array}
$$

where $I_{\lambda}(s, \vec{\Omega})$ is the spectral intensity at a given position $s$ and $I_{b \lambda}(T(s))$ is the blackbody intensity at temperature $T$ (a term involved in the classical formulation of the RTE that could be omitted in the present case since a cold medium is considered). $\kappa_{c \lambda}, \kappa_{d \lambda}, \sigma_{d \lambda}$ and $P_{\lambda}\left(\overrightarrow{\Omega^{\prime}} \rightarrow \vec{\Omega}\right)$ are the radiative properties of the medium, respectively corresponding to the gas phase and droplet spectral absorption coefficients, the droplet spectral scattering coefficient and the spectral scattering phase function.

- A DOM scheme as described in Collin et al. [11] is used, with a quadrature based on a split of the polar angle according to 146 directions with a non regular mesh providing an increasing accuracy in directions close to the main propagation direction. Namely, directions are separated by $0.05^{\circ}$ in the area between the axis and a polar angle of $5^{\circ}$, then by $2.5^{\circ}$ in the polar angle range from 5 to $60^{\circ}$ and finally by $5^{\circ}$ in the rest of the domain (recall that azimuthal symmetry is considered, avoiding the need for a discretization according to the azimuth). The diamond differencing scheme is used. The spray width is split into 30 regular meshes.

- Radiative properties are computed from the Mie theory in what concerns the part due to the water droplets (which are assumed to be perfectly spherical with constant size) and from a correlated-k model for the part related to the gaseous phase. Hypothesis of independent scattering is undoubtedly valid owing to the weak value of the droplet volumetric fraction (of the order of $10^{-5}$ ). A direct implementation of the Mie theory is used to evaluate the absorption and scattering coefficients and the scattering phase function. A renormalization of this last radiative property is then performed after the definition of the quadrature governing the directional discretization to ensure the conservation of the radiative energy. The c-k model as presented by Soufiani and Taine [14] is used with a spectral discretization according to 367 bands (which corresponds to a band width as expressed in wavenumber of $\left.25 \mathrm{~cm}^{-1}\right)$. 
On the basis of the predicted intensity distribution, the transmissivity is computed as

$$
\operatorname{Tr}_{\lambda}=\frac{\int_{\Omega_{\mathrm{det}}} I_{\lambda}^{\text {spray on }}(L, \vec{\Omega}) \cdot \cos \theta \cdot d \Omega}{\int_{\Omega_{\mathrm{det}}} I_{\lambda}^{\text {spray off }}(L, \vec{\Omega}) \cdot \cos \theta \cdot d \Omega}
$$

where $I_{\lambda}^{\text {spray on }}(L, \vec{\Omega})$ and $I_{\lambda}^{\text {spray off }}(L, \vec{\Omega})$ respectively stand for the intensity leaving the spray on the side opposite to the radiation source when the spray is on and when the spray is off (no droplets). $\theta$ is the polar angle defined between the propagation direction $\vec{\Omega}$ and the propagation axis. $\Omega_{\mathrm{det}}$ is a realistic solid angle value evaluated on the experimental facility for the detector angular acceptance. This angle condition is satisfied in solely considering the intensities along two directions around the axis which leads to a $0.1^{\circ}$ half angle of detection and so to a $0.2^{\circ}$ total angle angle close to the $0.17^{\circ}$ experimental value. Incident radiation is contained in an angle equal to $0.2^{\circ}$ around the axis, so along four of the discrete angles used in the simulation.

Numerical results as compared with corresponding experimental data are shown in figure 9 . A very good quantitative and qualitative agreement is found between experimental measurements and numerical predictions. The fact that the prediction slightly overpredicts the experimental data is in accordance with the observations discussed on Figure 6, when varying the lateral position of the detector. The transmissivity has been found to increase when shifting from the central position as a consequence of a decreasing volume fraction. The numerical computations involve an average volume fraction for the whole spray, which is lower than the actual value crossed by the beam near the spray centre, thus explaining a logical lower predicted attenuation (or higher transmission level). Anyway, the quantitative agreement achieved for the attenuation is not the very important feature we want to emphasize here, as this level is very dependent on parameters that have been evaluated with care but are not exactly known, mainly the exact size distribution of droplets (that controls the mean level of the transmission curve) and the curtain width or the moisture (that are directly involved in the absorption bands due to water vapor). On the other hand an undeniably good qualitative agreement is obtained with mainly the prediction of the slightly sloping, rather linear, variations of the transmissivity that increases with the wavenumber (outside vapor absorption bands). This phenomenon can be related to scattering because as the wavenumber increases, the size parameter of droplets $(\pi d / \lambda$ where $d$ is the droplet diameter and $\lambda$ the wavelength) increases and the scattering phase function becomes sharper. So a larger part of the scattered intensity remains in the solid angle of detection instead of leaving it and being lost.

[Figure 9 about here.]

[Figure 10 about here.] 
Numerical results produced by simulations omitting scattering (purely absorbant medium) and with scattering but with (complete simulation) or without scattering reinforcement are also presented in figure 10. In this last case, a null scattering coefficient is introduced for the scattering reinforcement integral term in the RTE (eq. 2). It is equivalent to consider an infinitely small angle of detection. The predicted transmission level is the smallest or the attenuation the largest, since the scattered radiation is lost. Note that this curve is also really flatter than the others despite obvious spectral variations of the radiative properties as reported in [12] for example. Actually increase in the scattering coefficient is balanced by decrease in the absorption coefficient and reciprocally, leading to a near constant level for the extinction coefficient. As scattering reinforcement is not addressed here, this results in a constant attenuation level outside of the water vapor absorption bands. On the contrary, transmissivity is the largest (attenuation the smallest) in the purely absorbant medium as there is no energy loss by scattering. This would be also the transmissivity obtained when considering a diffuse illumination of the spray and an hemispherical detection (not shown here), since scattering is very forward oriented so that the very most of the energy would be finally transmitted through the spray, scattering gains balancing losses. Finally an intermediate result is provided by the complete simulation when one considers the actual collimated illumination and detection angles. The largest the wavenumber is (or the smallest the wavelength is) the largest the reinforcement by scattering is. Despite the fact that radiation is only weakly deviated by scattering (as seen in the experimental result section) it has an actual role in the radiation attenuation. Note that this role could be less influent if larger incident and detection angles were considered. A last comment can be done regarding the curve referred as "model using a monodispersion" based on a pulverization of droplets with diameter equal to the mean Sauter diameter $(182 \mu \mathrm{m})$. The computation has been carried out considering the measured volumetric fraction and thus introducing a droplet density number of $1.85 \times 10^{7}$ droplets $/ \mathrm{m}^{3}$. Actually, the obtained result is close to the complete polydispersion one. A slight discrepancy may be observed, which can be attributed to scattering phenomena. Scattering reinforcement is obviously not exactly captured with the monodispersion simulation, even if the observed error remains very small. Note also that the monodispersion curve exhibits small oscillations that are due to interference patterns also observable on the radiative properties and that are smoothed when considering different droplet diameters. In any case, the possibility of using a representative monodispersion in order to characterize the spray behavior has to be kept in mind (discrepancy in the transmission prediction being less than $0.2 \%$ ) owing to the time that it could save, especially if a first stage of droplet tracking has to be performed in order to characterize the dynamics of the spray before considering the radiative part of the problem, a complete spray simulation which is in course in our laboratory. 


\section{Concluding remarks}

An experimental setup has been built and tested in order to study the ability of given water sprays to attenuate radiation. Transmission measurements performed for various water flow rate and at different vertical position from the nozzle exit have been reported on a nozzle referred as Tee Jet 400067, indicating that:

- transmissivity exhibits a rather flat mean level, with a slight slope however as the wavenumber rises and with sharp local variations in defined bands related to water vapor radiative properties,

- the vertical position in the spray seems to have little effect on the attenuation ability of the spray despite possible variation of the other spray characteristics (broadening, droplet diameter change, velocity alteration,...) thus expected to have compensating effects,

- on the contrary, a shift in the lateral position when recording the transmission data, results in a modification of the attenuation, as a consequence of inhomogeneities in the spray in the present case of a single nozzle device,

- average transmissivity first decreases as the water flow rate increases, but does not fall below a minimum level reached around a feed pressure of 4 bars, as a consequence of broadening of the spray which balances a larger flow rate (note that such observation would probably not hold in case of a multiple nozzle device owing to an expected overlapping of the sprays),

- numerical simulations have been performed in conditions close to the experimental ones indicating the ability of the code to reproduce the experimental measurements, provided a special care is brought to the sharp spectral variations (through a c-K model in the present study) and even more to the acute forward scattering behavior of the dispersed phase which has been captured here using a specific angular quadrature.

\section{Acknowledgement}

This work has been carried out in the frame of a scientific collaboration with the VKI (Von Karman Institute - Brussels). Pr Buchlin and M.R. Vetrano are fully acknowledged for their advice and comments when preparing the experimental facility and for the nozzles they put to our disposal. 


\section{References}

[1] H. Prétrel. Étude du comportement thermodynamique de pulvérisations liquides sous l'effet du rayonnement infrarouge. Application à la protection incendie par rideau d'eau. PhD thesis, INSA de Lyon, 1997.

[2] S. Dembélé, J.X. Wen, and J.F. Sacadura. Experimental study of water sprays for the attenuation of fire thermal radiation. ASME J. Heat Transfer, 123:534-543, 2001.

[3] J.V. Murrell, D. Crowhurst, and P. Rock. Experimental study of the thermal radiation attenuation of sprays from selected hydraulic nozzles. In Proceedings of Halon Options Technical Working Conference 95, pages 369-378, 1995.

[4] K. Hald and J.M. Buchlin. Thermal shielding by impinging water spray curtains. In Proceedings of Eurotherm 73 on Computational Thermal Radiation in Participating Media, Mons, pages 379-387, 2003.

[5] J.F. Widmann and J. Duchez. The effect of water sprays on fire fighter thermal imagers. Fire Safety Journal, 39:217-238, 2004.

[6] G. Grant, J. Brenton, and D. Drysdale. Fire suppression by water sprays. Progress in Energy and Combustion Science, 26:79-130, 2000.

[7] J.F. Sacadura. Radiative transfer in fire safety science. In Proceedings of the 4th International Symposium on Radiative Transfer, Istanbul, Turkey, pages 3-18, 2004.

[8] W. Grosshandler, D. Lowe, K. Notarianni, and W. Rinkinen. Protection of data processing equipment with fi ne water sprays. NIST report, $\mathrm{n}^{\circ}$ NISTIR 5514, 1994.

[9] L.Zimmer, P. Boulet, and J.M. Buchlin. Experimental and numerical study of flat fan sprays under lateral wind. In Proceedings of the 4th International Conference on Multiphase Flow, New Orleans, USA, page paper 176, 2001.

[10] S. Dembélé. Modélisation et étude expérimentale des transferts de chaleur par rayonnement dans un rideau d'eau diphasique. Application à la protection incendie d'installations industrielles à risques. PhD thesis, INSA de Lyon, 1998.

[11] A. Collin, P. Boulet, D. Lacroix, and G. Jeandel. On radiative transfer in water spray curtains using the discrete ordinates method. J. Quant. Spect. Rad. Tran., Accepted, in press. 
[12] N. Berour, D. Lacroix, P. Boulet, and G. Jeandel. Radiative and conductive heat transfer in a non-grey semitransparent medium - application to fire protection curtains. J. Quant. Spect. Rad. Tran., 86:9-30, 2004.

[13] L. Zimmer. Etude numérique et expérimentale de la turbulence en écoulement gaz-gouttelettes. Applications aux rideaux d'eau en présence de vent latéral. PhD thesis, Institut Von Karman et UHP Nancy 1, 2001.

[14] A. Soufiani and J. Taine. High temperature gas radiative property parameters of statistical narrow-band model for $\mathrm{H}_{2} \mathrm{O}, \mathrm{CO}_{2}$ and $\mathrm{CO}$, and correlated-k model for $\mathrm{H}_{2} \mathrm{O}$ and $\mathrm{CO}_{2}$. Int. J. Heat Mass Transfer, 40(4):987-991, 1997. 


\section{Nomenclature}

- $d$, droplet diameter (m)

- $f_{v}$, droplet volume fraction $\left(\mathrm{m}^{3}\right.$ of water $/ \mathrm{m}^{3}$ of air)

- $I_{\lambda}^{0}(T)$, blackbody spectral intensity at temperature $T\left(\mathrm{~W} \cdot \mathrm{m}^{-3} \cdot \mathrm{sr}^{-1}\right)$

- $I_{\lambda}$, spectral intensity $\left(\mathrm{W} \cdot \mathrm{m}^{-3} \cdot \mathrm{sr}^{-1}\right)$

- $L$, width of the medium (m)

- $P_{\lambda}$, scattering phase function

- $s$, position in the medium (m)

- $T$, temperature $(\mathrm{K})$

- $T r$, transmissivity

Greek symbols

- $\kappa$, absorption coefficient $\left(\mathrm{m}^{-1}\right)$

- $\sigma$, scattering coefficient $\left(\mathrm{m}^{-1}\right)$

- $\theta$, polar angle (rad)

- $\Omega$, solid angle (sr)

- $\vec{\Omega}$, propagation direction

Subscripts

- $c$, continuous phase property

- $d$, droplet property

- det, detector characteristic

- $\lambda$, spectral property

- $\nu$, wavenumber dependent property 


\section{List of Figures}

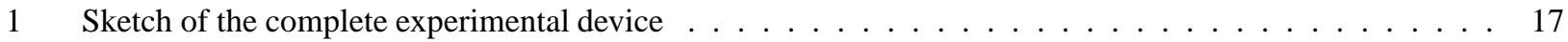

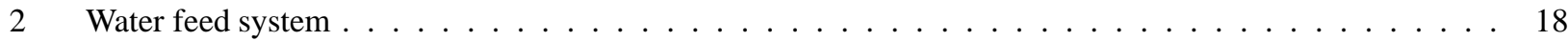

3 Water flow versus water pressure . . . . . . . . . . . . . . . . . . . . . . . . . 19

4 Spectral variations of the transmissivity. Vertical position $h$ is $20 \mathrm{~cm}$, lateral position is $0 \mathrm{~cm} \ldots \ldots$. . 20

5 Variations of the average transmissivity versus water pressure. Lateral position is $0 \mathrm{~cm} \ldots \ldots 21$

6 Variations of the average transmissivity versus pressure for various lateral positions $x$. Vertical position $h$

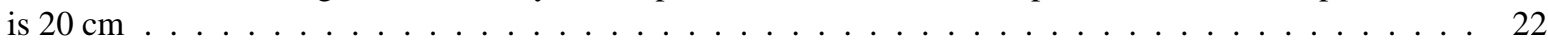

7 Normalized angular variations of the intensity detected with the spray (water pressure: 4 bars, vertical

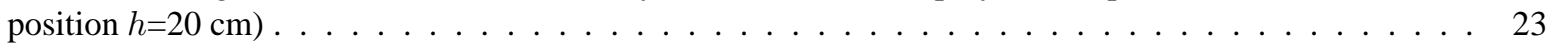

8 Normalized intensity with spray on and with spray off as a function of detection angle at the wavenumber

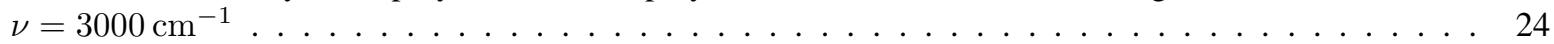

9 Experimental and numerical transmissivity. Water pressure is $1,5 \mathrm{bar}$, vertical position is $20 \mathrm{~cm}$, lateral

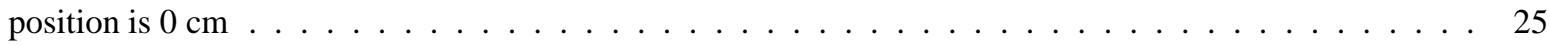

10 Numerical transmissivity obtained with various numerical parameters. Water pressure is $1,5 \mathrm{bar}$, vertical

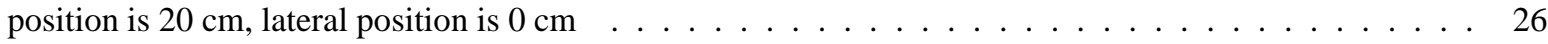




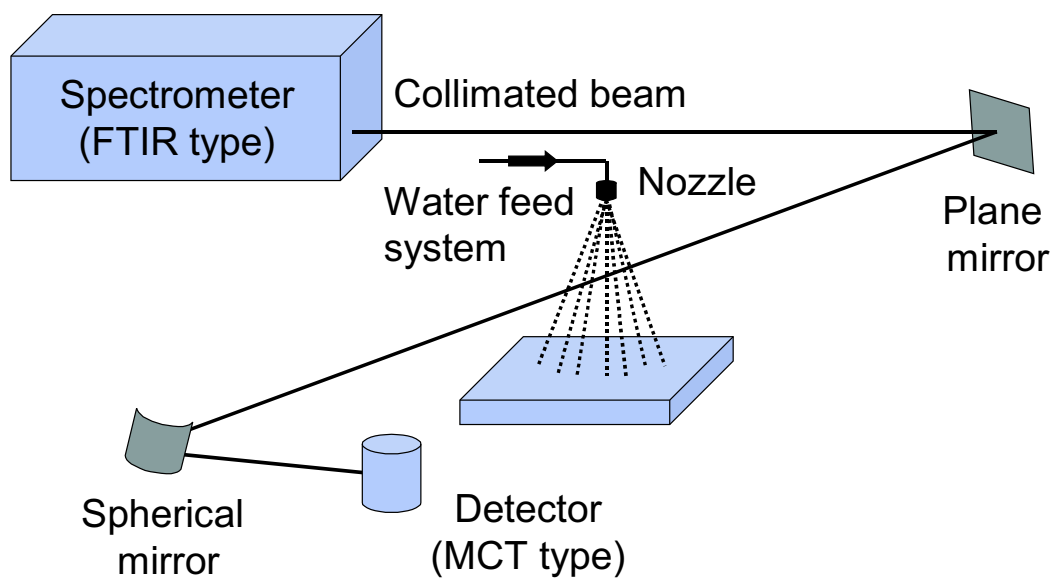

Figure 1: Sketch of the complete experimental device 


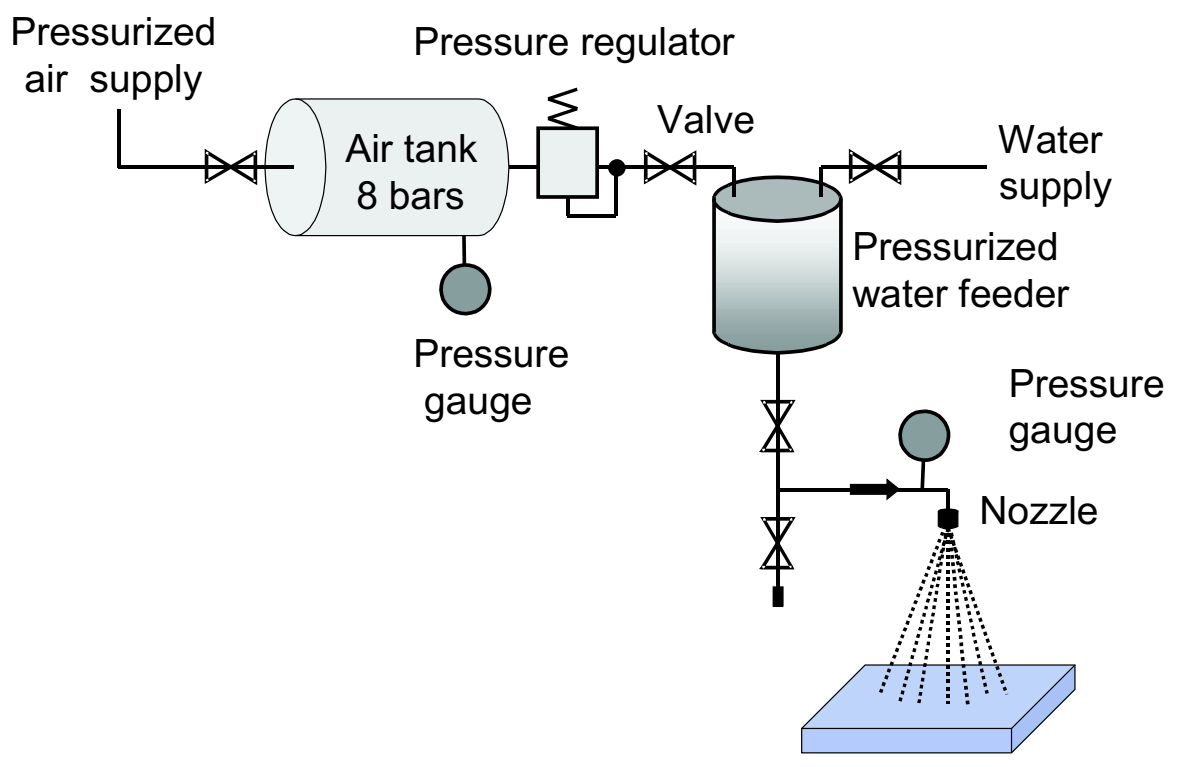

Figure 2: Water feed system 


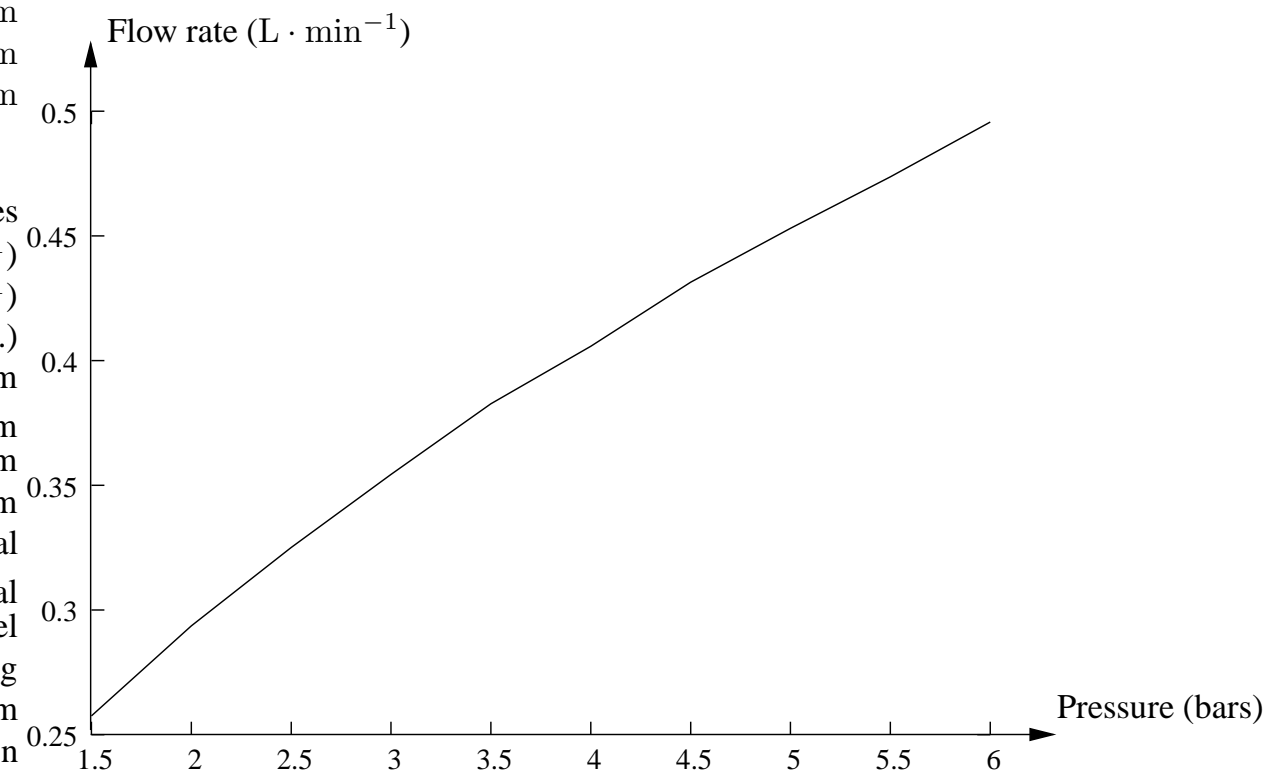

Figure 3: Water flow versus water pressure 


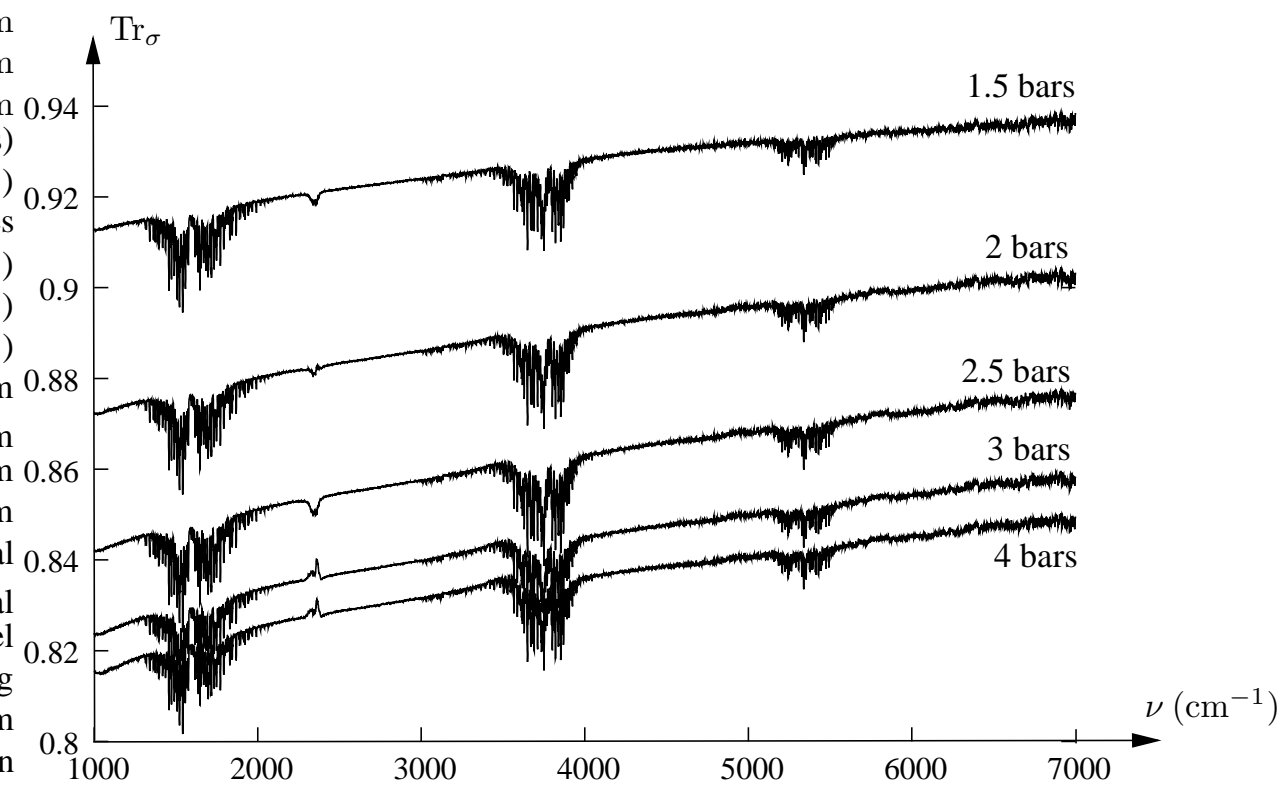

Figure 4: Spectral variations of the transmissivity. Vertical position $h$ is $20 \mathrm{~cm}$, lateral position is $0 \mathrm{~cm}$ 


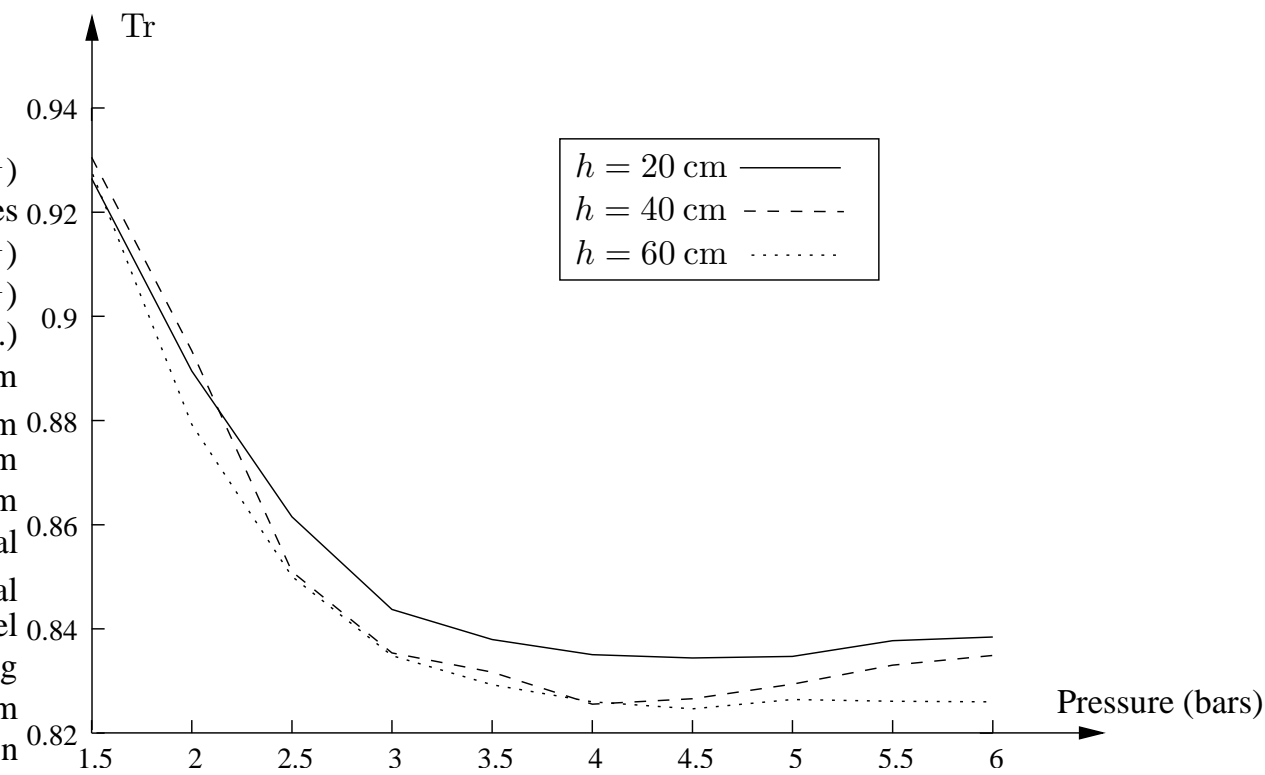

Figure 5: Variations of the average transmissivity versus water pressure. Lateral position is $0 \mathrm{~cm}$ 


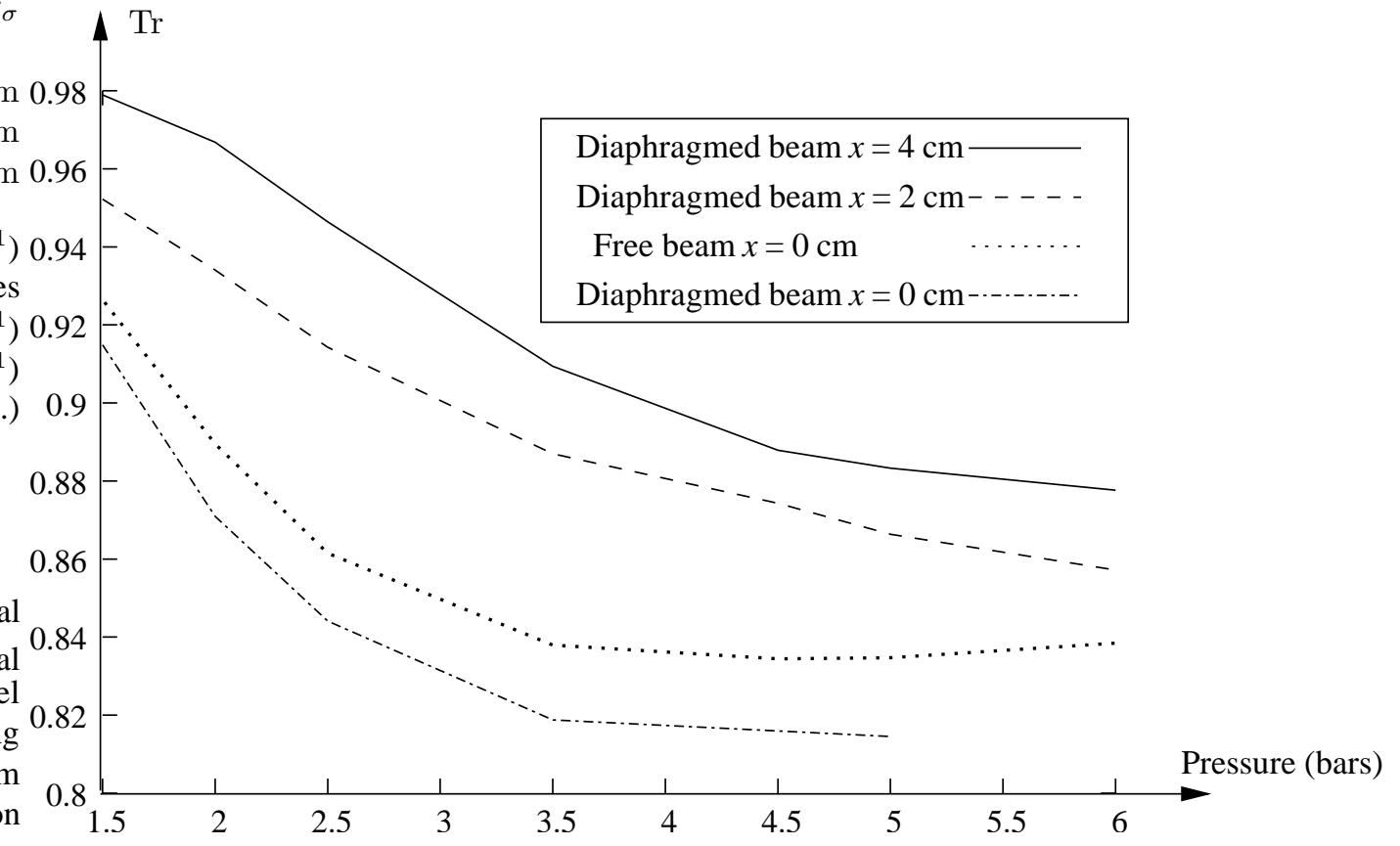

Figure 6: Variations of the average transmissivity versus pressure for various lateral positions $x$. Vertical position $h$ is 20 $\mathrm{cm}$ 


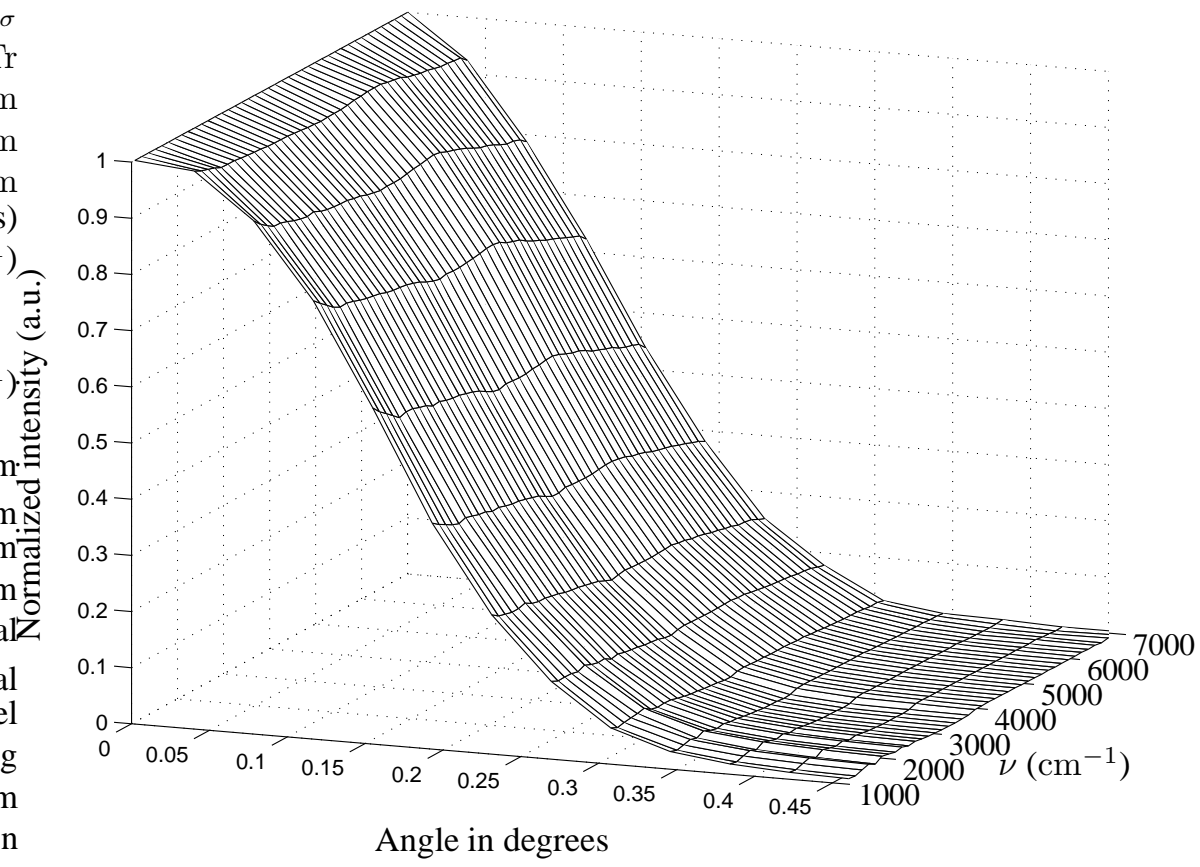

Figure 7: Normalized angular variations of the intensity detected with the spray (water pressure: 4 bars, vertical position $h=20 \mathrm{~cm})$ 


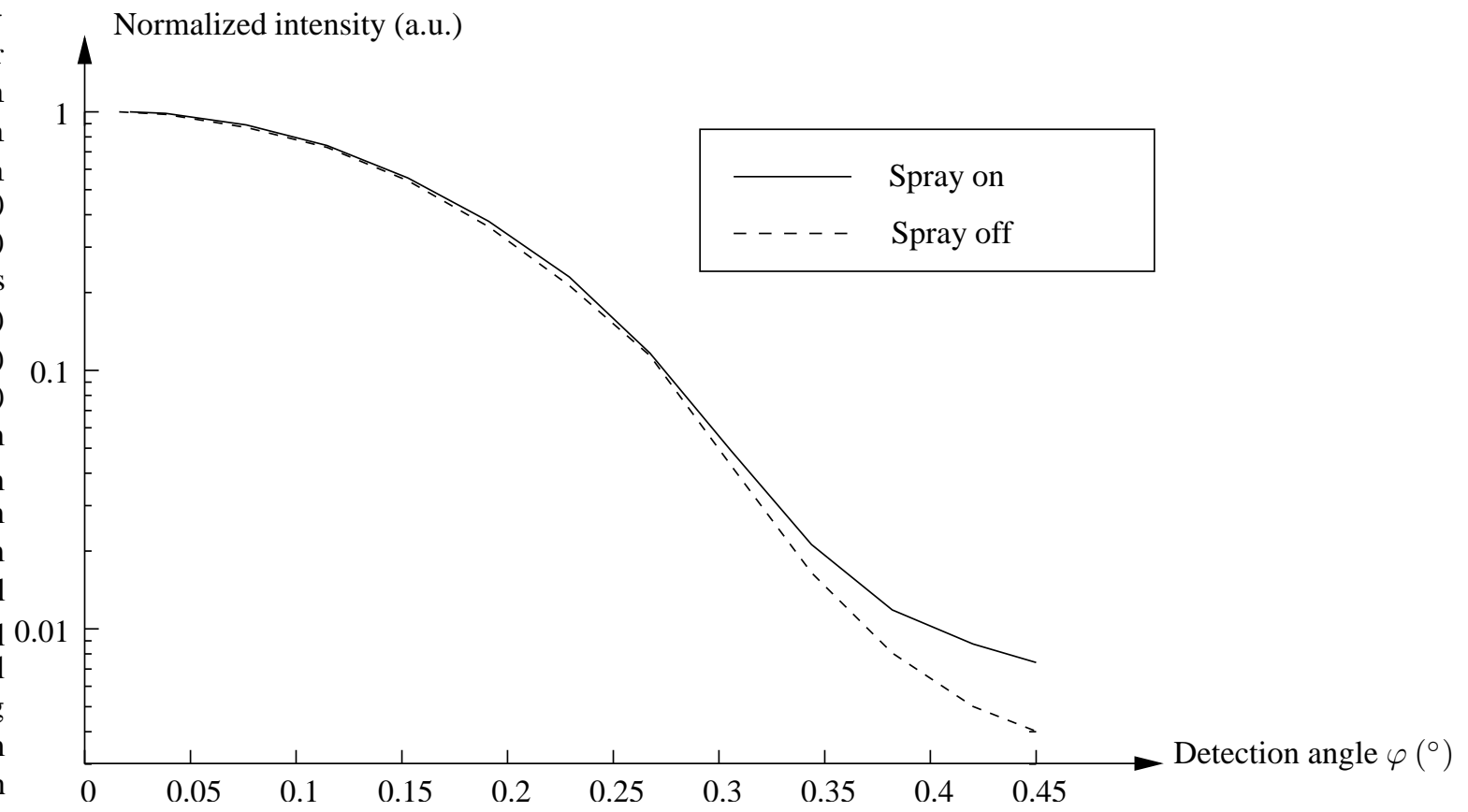

Figure 8: Normalized intensity with spray on and with spray off as a function of detection angle at the wavenumber $\nu=3000 \mathrm{~cm}^{-1}$ 


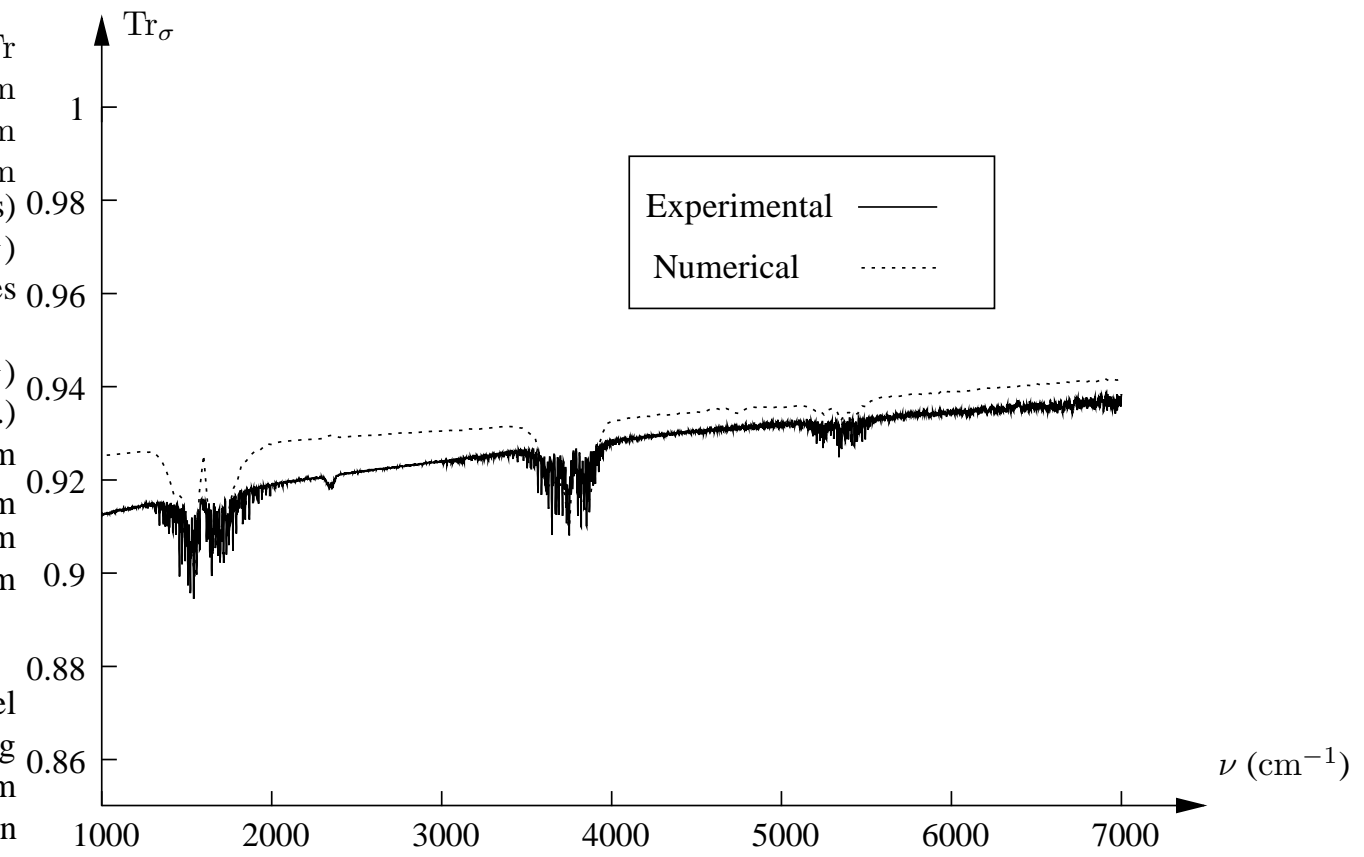

Figure 9: Experimental and numerical transmissivity. Water pressure is $1,5 \mathrm{bar}$, vertical position is $20 \mathrm{~cm}$, lateral position is $0 \mathrm{~cm}$ 


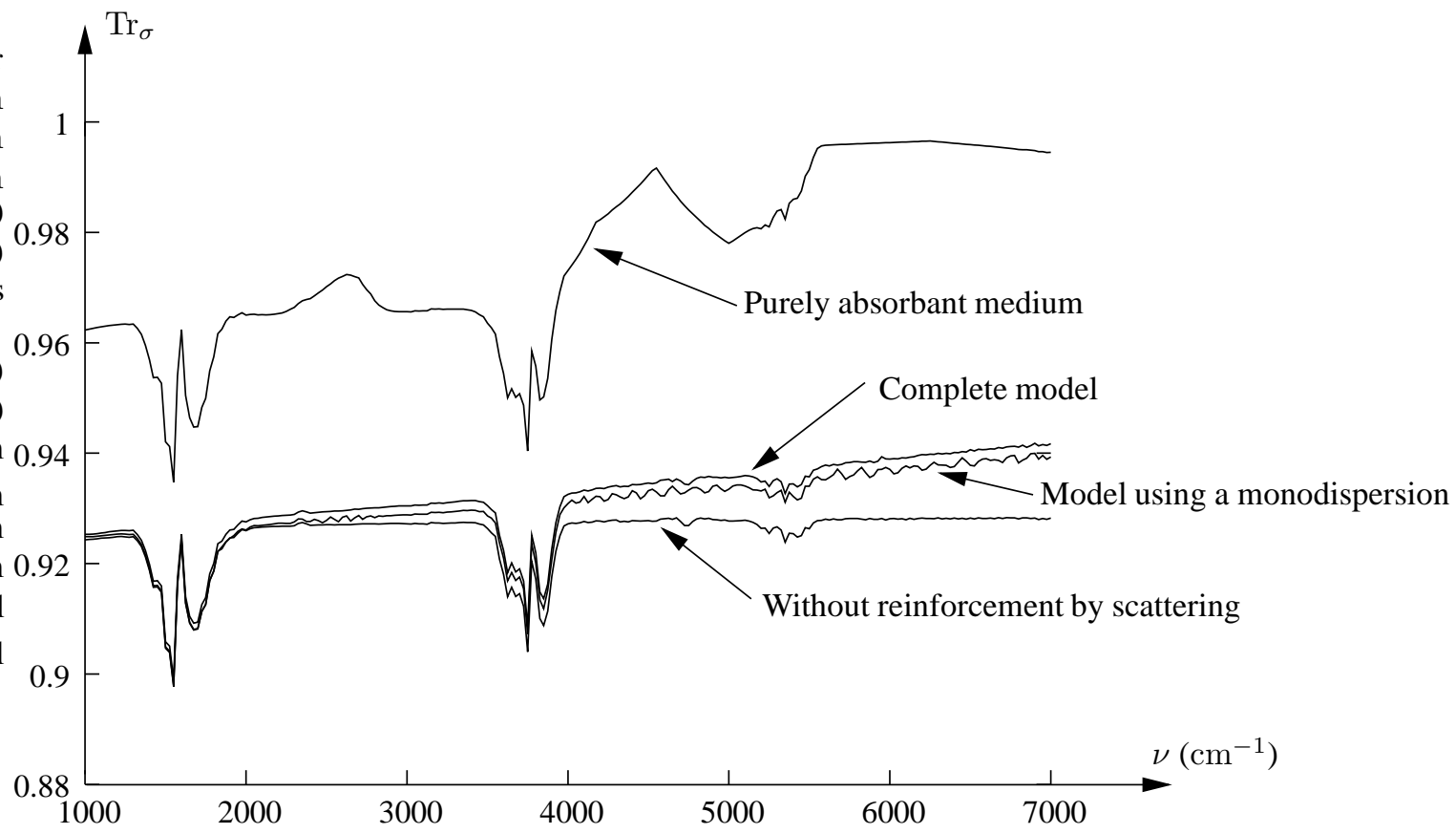

Figure 10: Numerical transmissivity obtained with various numerical parameters. Water pressure is 1,5 bar, vertical position is $20 \mathrm{~cm}$, lateral position is $0 \mathrm{~cm}$ 